\section{The Author's Reply: Sleep Complaints in \\ Hospitalized Italian Patients with Hematological Malignancies}

Key words: insomnia, depression, hematological disorders

\section{(Inter Med 49: 1691, 2010)}

(DOI: 10.2169/internalmedicine.49.3914)

We thank Dr Andrea and colleagues for their interest and comments on our paper (1), which have provided us with the opportunity to clarify some aspects of our report.

For patients with cancer, sleep is potentially affected by a variety of factors, including environmental factors, such as isolation, biochemical changes associated with the process of neoplastic growth and anticancer treatments, and symptoms that frequently accompany cancer, such as pain, fatigue, and depression. The rate of insomnia in cancer patients exceeds that in the general population and varies according to the definition used to make the diagnosis. The percentage of patients meeting formal criteria for an insomnia disorder has been reported at $19 \%$, and that of patients self-reporting disturbed sleep has been reported as high as $63 \%$ (2). Our study was retrospective, and the detailed medical history and patient information were extracted from patients' charts and medical records at the hospital. We assessed insomnia based on individual symptoms, including difficulty initiating sleep, difficulty maintaining sleep, early morning awakening, and requests for hypnotics. Approxi- mately $25 \%$ to $50 \%$ of all prescriptions written for patients with cancer are for hypnotics $(3,4)$. We could not compare our results with previous reports due to differences in the study design and background of patients.

We agree that larger studies are needed to assess insomnia risk factors for patients with hematological disorders. Ideally, well-designed prospective and multi-institution studies including fixed scales and assessments by a psychiatrist are needed to accurately evaluate insomnia.

Akane Kunitomi ${ }^{1,2}$, Hiroatsu Iida ${ }^{1}$, Yoshikazu Kamiya ${ }^{1}$,

Mayuko Hayashi ${ }^{1}$, Taro Suwa ${ }^{3}$, Junya Kanda ${ }^{4}$ and

Hiroshi Sao ${ }^{1}$

\section{References}

1. Kunitomi A, Iida H, Kamiya $\mathrm{Y}$, et al. Insomnia and depression during protective isolation in patients with hematological disorders. Intern Med 49: 17-22, 2010.

2. Stepanski EJ, Walker MS, Schwartzberg LS, Blakely LJ, Ong JC, Houts AC. The relation of trouble sleeping, depressed mood, pain, and fatigue in patients with cancer. J Clin Sleep Med 15: 132-136, 2009.

3. Palesh OG, Roscoe JA, Mustian KM, et al. Prevalence, demographics, and psychological associations of sleep disruption in patients with cancer: University of Rochester Cancer CenterCommunity Clinical Oncology Program. J Clin Oncol 28: 292298, 2010.

4. Roscoe JA, Kaufman ME, Matteson-Rusby SE, et al. Cancerrelated fatigue and sleep disorders. Oncologist 12(Suppl 1): 35-42, 2007.

\footnotetext{
${ }^{1}$ Department of Hematology, Meitetsu Hospital, Nagoya, ${ }^{2}$ Department of Hematology, Japanese Red Cross Society Wakayama Medical Center, Wakayama, ${ }^{3}$ Department of Psychiatry, Graduate School of Medicine, Kyoto University, Kyoto and ${ }^{4}$ Department of Hematology and Oncology, Graduate School of Medicine, Kyoto University, Kyoto

Received for publication May 6, 2010; Accepted for publication May 13, 2010

Correspondence to Dr. Akane Kunitomi, akunitom@mx5.suisui-w.ne.jp

(C) 2010 The Japanese Society of Internal Medicine Journal Website: http://www.naika.or.jp/imindex.html
} 